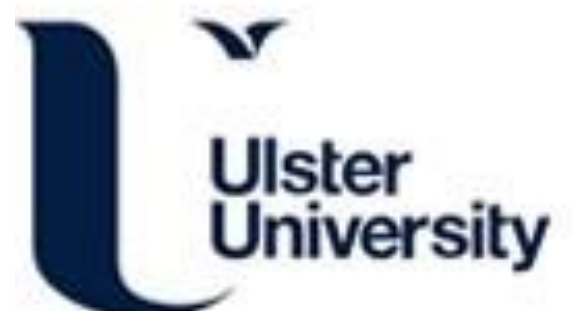

Tuning the Catalytic Activity of Graphene Nanosheets for Oxygen Reduction Reaction via Size and Thickness Reduction

Benson, J., Xu, Q., Wang, P., Shen, Y., Sun, L., Wang, T., Li, M., \& Papakonstantinou, P. (2014). Tuning the Catalytic Activity of Graphene Nanosheets for Oxygen Reduction Reaction via Size and Thickness Reduction. ACS Applied Materials and Interfaces, 6, 19726-19736. https://doi.org/10.1021/am5048202

Link to publication record in Ulster University Research Portal

Published in:

ACS Applied Materials and Interfaces

Publication Status:

Published (in print/issue): 21/10/2014

DOI:

10.1021/am5048202

Document Version

Publisher's PDF, also known as Version of record

\section{General rights}

Copyright for the publications made accessible via Ulster University's Research Portal is retained by the author(s) and / or other copyright owners and it is a condition of accessing these publications that users recognise and abide by the legal requirements associated with these rights.

\section{Take down policy}

The Research Portal is Ulster University's institutional repository that provides access to Ulster's research outputs. Every effort has been made to ensure that content in the Research Portal does not infringe any person's rights, or applicable UK laws. If you discover content in the Research Portal that you believe breaches copyright or violates any law, please contact pure-support@ulster.ac.uk. 


\title{
Tuning the Catalytic Activity of Graphene Nanosheets for Oxygen Reduction Reaction via Size and Thickness Reduction
}

\author{
John Benson, ${ }^{\dagger}$ Qian Xu, ${ }^{\ddagger}$ Peng Wang, ${ }^{\ddagger}$ Yuting Shen, ${ }^{\S}$ Litao Sun, ${ }^{\S}$ Tanyuan Wang, " Meixian Li, \\ and Pagona Papakonstantinou ${ }^{*}$, \\ ${ }^{\dagger}$ Engineering Research Institute, School of Engineering, University of Ulster, Newtownabbey BT37 0QB, United Kingdom \\ ${ }^{ \pm}$National Laboratory of Solid State Microstructures and College of Engineering and Applied Sciences, Nanjing University, 22 Hankou \\ Road, Gulou, Nanjing 210093, People's Republic of China \\ ${ }^{\S}$ SEU-FEI Nano-Pico Center, Key Laboratory of MEMS of Ministry of Education, Southeast University, Sipailou 2, Nanjing 210096, \\ People's Republic of China \\ "College of Chemistry and Molecular Engineering, Peking University, Beijing 100871, People’s Republic of China
}

\section{Supporting Information}

ABSTRACT: Currently, the fundamental factors that control the oxygen reduction reaction (ORR) activity of graphene itself, in particular, the dependence of the ORR activity on the number of exposed edge sites remain elusive, mainly due to limited synthesis routes of achieving small size graphene. In this work, the synthesis of low oxygen content $(<2.5 \pm 0.2$ at. $\%)$, few layer graphene nanosheets with lateral dimensions smaller than a few hundred nanometers were achieved using a combination of ionic liquid assisted grinding of high purity

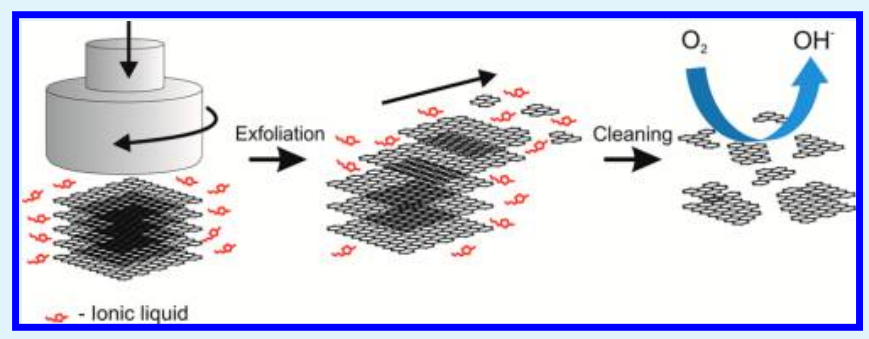
graphite coupled with sequential centrifugation. We show for the first time that the graphene nanosheets possessing a plethora of edges exhibited considerably higher electron transfer numbers compared to the thicker graphene nanoplatelets. This enhanced ORR activity was accomplished by successfully exploiting the plethora of edges of the nanosized graphene as well as the efficient electron communication between the active edge sites and the electrode substrate. The graphene nanosheets were characterized by an onset potential of $-0.13 \mathrm{~V}$ vs $\mathrm{Ag} / \mathrm{AgCl}$ and a current density of $-3.85 \mathrm{~mA} / \mathrm{cm}^{2}$ at $-1 \mathrm{~V}$, which represent the best $\mathrm{ORR}$ performance ever achieved from an undoped carbon based catalyst. This work demonstrates how low oxygen content nanosized graphene synthesized by a simple route can considerably impact the ORR catalytic activity and hence it is of significance in designing and optimizing advanced metal-free ORR electrocatalysts.

KEYWORDS: graphene nanosheets, oxygen reduction reaction, electrocatalyst, edges, ionic liquid exfoliation

\section{INTRODUCTION}

Increasing demands for clean energy have stimulated extensive research on the development of technologies that can effectively convert chemical energy into electricity with high efficiency and at low cost. Catalysts for the oxygen reduction reaction (ORR) are at the heart of key electrochemical technologies including low temperature polymer electrolyte membrane (PEM) fuel cells and metal air batteries. ${ }^{1-5}$ The existing catalysts of Pt group metals are highly efficient but too expensive and rare to be useful for mass production. ${ }^{6-8}$ Graphene promises a low-cost alternative to the precious metals. $^{9-12}$ In particular, there has been an explosion of studies on introducing various heteroatoms (e.g., N, B, P, S, and I) ${ }^{13-16}$ or a combination of those $\mathrm{e}^{17,18}$ into graphene demonstrating a competitive ORR activity compared to the benchmark Pt catalyst. The phenomenon has been rationalized using density functional calculations, where it was found that the heteroatom induces an uneven charge distribution in the adjacent sites, which alters the local spin or charge density. This promotes $\mathrm{O}$ adsorption and facilitates efficient ORR performance. $^{19,20}$

However, despite the remarkable progress, fundamental factors that control the ORR activity of graphene itself, in particular, the dependence of the ORR activity on the number of exposed edge sites remains elusive due to limited synthesis routes of achieving small size and preferably oxygen free graphene sheets. For large graphene sheets, an altered electronic structure is expected at the basal plane, as compared to the edge region, due to symmetry breaking of the honeycomb lattice. Graphene has two main kinds of edge terminations according to their shape, named zigzag and armchair edges. These two edges have different electronic structures. According to theoretical and experimental studies $^{21,22}$ a $\pi$-electron state called edge state is created along the zigzag edges, whereas no such state is present at the armchair

Received: July 22, 2014

Accepted: October 21, 2014 
edges. This characteristic edge state, which has a large local density of states and is spin polarized, gives rise to electronic, magnetic, and chemical activities in the zigzag edges of graphene. Recent experimental studies on large area graphene have provided supporting evidence for the high ORR activity of the edges. ${ }^{23}$ Hence, it can be insinuated that a prerequisite for a highly ORR electroactive graphene catalyst is the ability to nanostructure graphene in a manner conducive of maximizing the number of exposed edge sites (preferably zigzag) relative to catalytically inert basal planes sites. ${ }^{24-26}$

As far as we are aware, no attempt has been made to synthesize graphene sheets, simultaneously possessing low oxygen content and small lateral dimensions with the view of examining their electroactivity. It is worth noting that under ambient conditions, a low concentration of oxygen $(<3$ at. $\%$ depending on the amount of edges) will always be present, because open graphene edges can be easily terminated by oxygen groups. Carbon materials possessing oxygen species are known to favor the $2 \mathrm{e}^{-}$process in ORR. ${ }^{27}$ Moreover, progressive addition of oxygen content in carbon materials has been shown to limit and slow down the electron transfer reactions. ${ }^{28}$ Therefore, introducing purposely more oxygen in the graphene is not considered to have a beneficial effect for achieving efficient (toward $4 \mathrm{e}^{-}$) ORR.

Clearly, there remains a dearth of experimental data on the ORR activity of low oxygen level graphene nanosheets. Here we have developed a novel approach for the production of low oxygen content few layer graphene nanosheets via simple and green ionic liquid assisted mechanical exfoliation of graphite ${ }^{29}$ combined with gradient centrifugation steps. The grinding of graphite with a small quantity of ionic liquid produces a gel due to the $\pi-\pi$ interactions between the graphite and ionic liquid. During the grinding process the ionic liquid acts as a lubricant, allowing the breaking of graphite platelets to smaller sizes and at the same time helps exfoliation of graphite layers through shear forces exerted on the graphite flake. Although the bucky gels produced by grinding of carbon nanomaterials with ionic liquid have been known since $2003^{30}$ and they have had uses in a number of applications such as supercapacitors, biosensors, and actuators, ${ }^{31}$ to our knowledge, the production of nanosized graphene with a low amount of oxygen has not been reported. Using our process, defect formation on the crystalline plane of graphene, or chemical reactions due to mechanochemical effects are avoided, resulting in high quality material. The edges as well as the basal plane of the graphene nanosheets are free from any additional functional groups, possessing only a small amount of oxygen $(<2.5 \pm 0.2$ at. \%) mainly inherited from the starting graphite and exposure of edges to atmosphere.

The sequential centrifugation steps employed here help to isolate graphene nanosheets with small lateral dimensions. The process dramatically enhances the presence of small size graphene nanosheets, thus providing abundant catalytic sites. We have taken extensive measures to ensure the grinding method does not involve metal components unlike many ball milling techniques, and therefore metal contamination from the grinding instrument is avoided. ${ }^{32,33}$ As a result, this new synthesis approach is ideal for revealing and gaining more knowledge on the role of graphene edges for the ORR.

We show for the first time that the ORR activity of graphene nanosheets possessing a plethora of edges with limited amount of oxygen $(<2.5 \pm 0.2$ at. \%) is substantially improved when compared to thicker graphene nanoplatelets, as revealed by enhanced electron transfer numbers. Therefore, the current study of the ORR on low oxygen content graphene nanosheets is expected to provide new insight into the design and feasible synthesis of more advanced graphene based catalysts.

\section{RESULTS AND DISCUSSION}

In a typical experiment, grinding was carried out in a planetary grinding machine in the presence of high purity graphite ( $\geq 99.99 \%)$ and ionic liquid (IL, 1-butyl-3-methylimidazolium hexafluorophosphate, $\mathrm{BMIMPF}_{6}, \geq 97.0 \%$ ), as depicted in the schematic of Figure 1. After grinding, the produced gel was

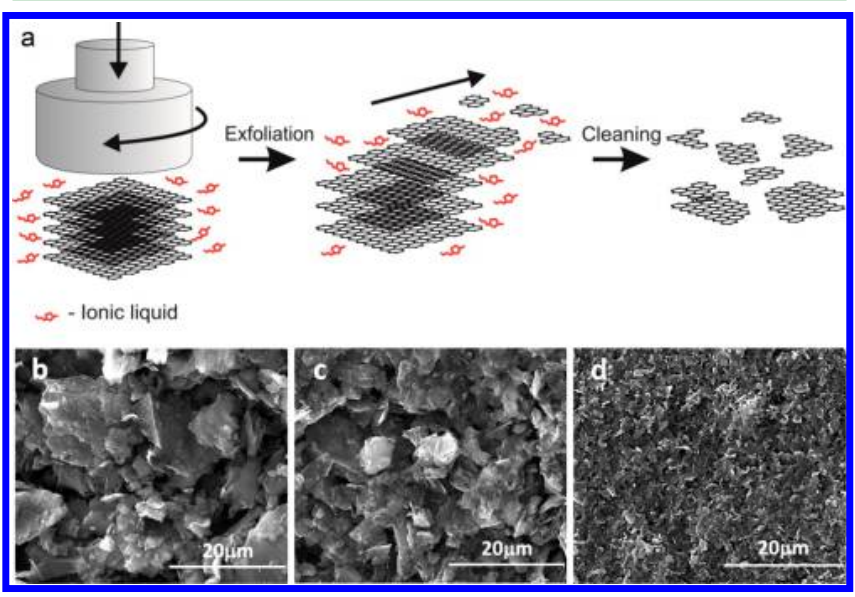

Figure 1. (a) Schematic representation of exfoliation of graphite. Shear forces exfoliate layers in a mechanical mortar grinder with ionic liquid, resulting in cleaned graphene nanosheets, free from functional groups. SEM images of (b) pristine graphite; sediment of ground material obtained at successive centrifugation speeds of (c) $1000 \mathrm{rpm}(1 \mathrm{~K})$ and (d) $10000 \mathrm{rpm}(10 \mathrm{~K})$.

washed in a mixture of dimethylformamide (DMF) and acetone to remove the ionic liquid. ${ }^{34}$ The supernatant from a DMF solution that contained the graphene nanosheets was collected through sequential centrifugation at 1000, 3000, and 10000 rpm speeds; ${ }^{35}$ for more information, see the Supporting Information. In this work, the products are named as XK for convenience, where $\mathrm{XK}$ denotes the centrifugation speed in thousands of rpm used to collect the sediment. The pristine graphite consists of large graphite platelets $(\sim 45 \mu \mathrm{m})$ as well as smaller irregular graphite flakes. Scanning electron microscopy (SEM) images of pristine graphite and graphene nanosheets produced through various centrifugation speeds at both lowand high magnification can be seen in Figures 1 and $S 1$ (Supporting Information). Grinding in ionic liquid coupled with sequential centrifugation gave rise to progressive size selection. Centrifugation at $10 \mathrm{~K}$ resulted in a dramatic decrease in sheet size $(<1 \mu \mathrm{m})$, (Figure S1, Supporting Information), exposing a large fraction of edge sites compared to lower centrifugation speeds.

High-resolution TEM (HRTEM) studies provided information on the crystalline quality of the sheets as well as their thickness. Characterization of the residue products from the sequential centrifugation steps by transmission electron microscopy (TEM) ensured that the final $10 \mathrm{~K}$ product consists of few layer sheets (up to 9) with overall lateral dimensions smaller than a few hundred nanometers, in contrast to those for $1 \mathrm{~K}$ and $3 \mathrm{~K}$, which were composed of thicker and larger size products, (Figures $2 \mathrm{a}-\mathrm{i}$ and $\mathrm{S} 2-\mathrm{S} 6$, Supporting Information). In fact, for the $10 \mathrm{~K}$ product, small size sheets are self-assembled onto the surface of larger few layer graphene sheets, most 


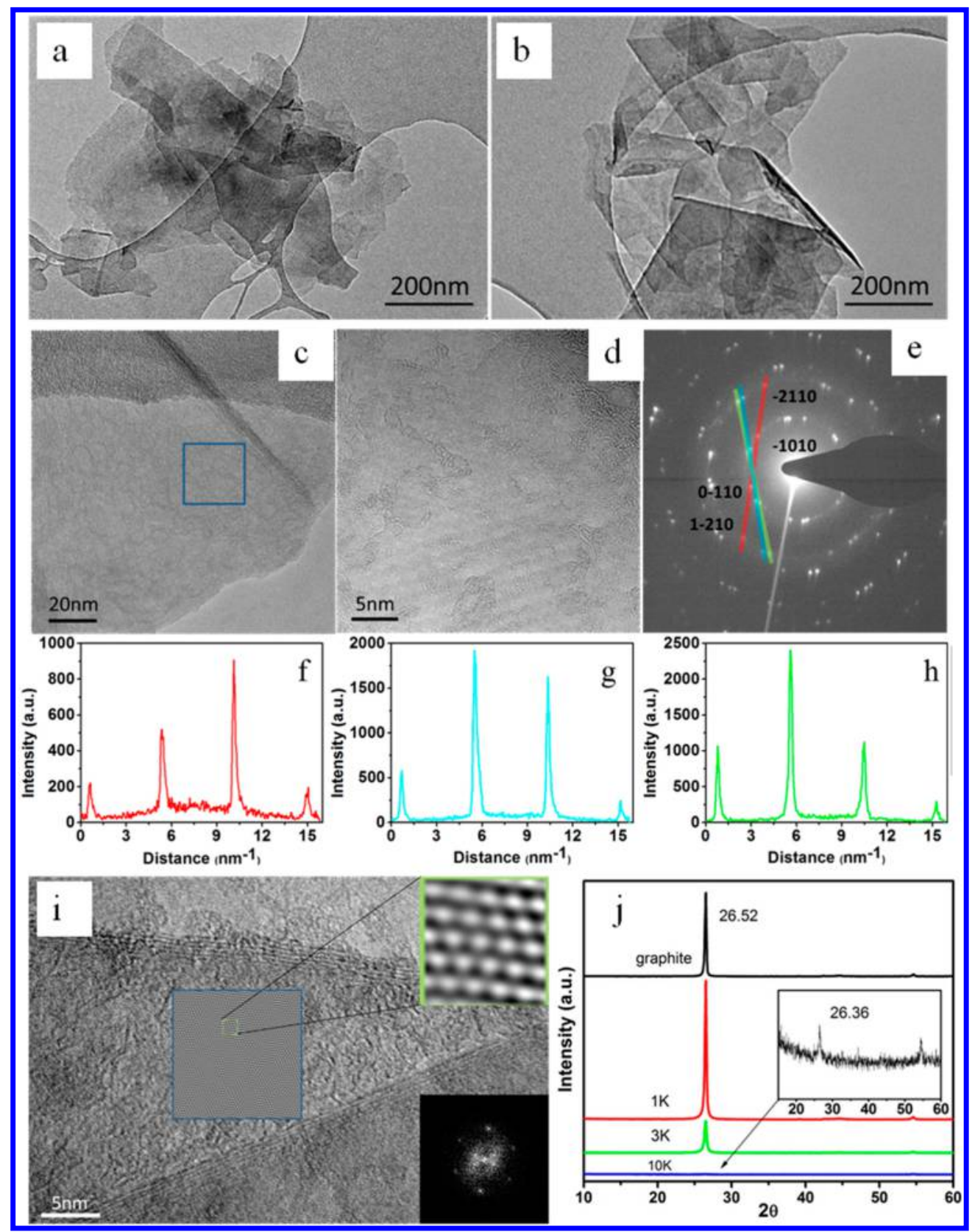

Figure 2. TEM images of typical products found in the $10 \mathrm{~K}$ sediment. $(\mathrm{a}, \mathrm{b}) 10 \mathrm{~K}$ product is dominated by nanosized few layer graphene assembled on larger sheets. (c) Low magnification TEM images from $10 \mathrm{~K}$ product acquired on an aberration correction Titan $80-300$ operated at $80 \mathrm{kV}$, with (d) magnified region of the image in panel c. (e) Corresponding electron diffraction pattern of panel d, which indicates that there are at least three different orientations of graphene. ( $\mathrm{f}-\mathrm{h}$ ) Intensity profile of diffracted spot extracted along the -2110 to $1-210$ axis for three oriented patterns in panel e. Ratios of the intensity of $\{1100\}$ and $\{2110\}$ are all $>1$, which indicates that the graphene in each orientation is formed with a single layer. (i) HRTEM of a 4-5 layer graphene: (top right corner) an enlargement of the noise-filtered area as marked with a blue square, where the clearly visible parallel lines demonstrate the regular period of the lattice planes; (bottom right corner) a Fourier transform of the blue marked area of the raw image. (j) X-ray diffraction (XRD) diffraction patterns graphite together with those of $1 \mathrm{~K}, 3 \mathrm{~K}$, and 10K products. Inset shows magnified section of $10 \mathrm{~K}$ product. Peak intensity (002) is $0.5 \%$ of pristine graphite, indicating a high degree of exfoliation and a decrease in lateral dimensions.

probably due to $\pi-\pi$ interactions. Such a self-assembled configuration allows more efficient transport. It is apparent that the nanosheets have a flat morphology, different to that reported $^{36}$ for reduced graphene oxide with the partially crumbled nature that originates from the defective structure formed during the fabrication of graphene oxide.

HRTEM image performed on one typical cross-section edge of the nanosheet, indicates an interlayer spacing of $0.326 \mathrm{~nm}$ for (001) plane, (Figure S5, Supporting Information). Selected area electron diffraction (SAED) pattern from a $10 \mathrm{~K}$ nanosheet about 3-4 layers reveals at least three sets of hexagonal patterns, indicating different orientations. Computational and experimental studies ${ }^{37-39}$ have shown that TEM SAED can convincingly distinguish monolayer from multilayer graphene by comparing the intensity ratio of first-order to second-order diffraction spots and diffraction peak intensity variation with respect to the tilt angle of the sample holder. It was observed that multilayer graphene with Bernal $(\mathrm{AB})$ stacking gives higher diffracted intensities for the outer $\{2110\}$-type spots than inner $\{1100\}$-type spots $(I\{1100\} / I\{2100\}<1)$, because of 


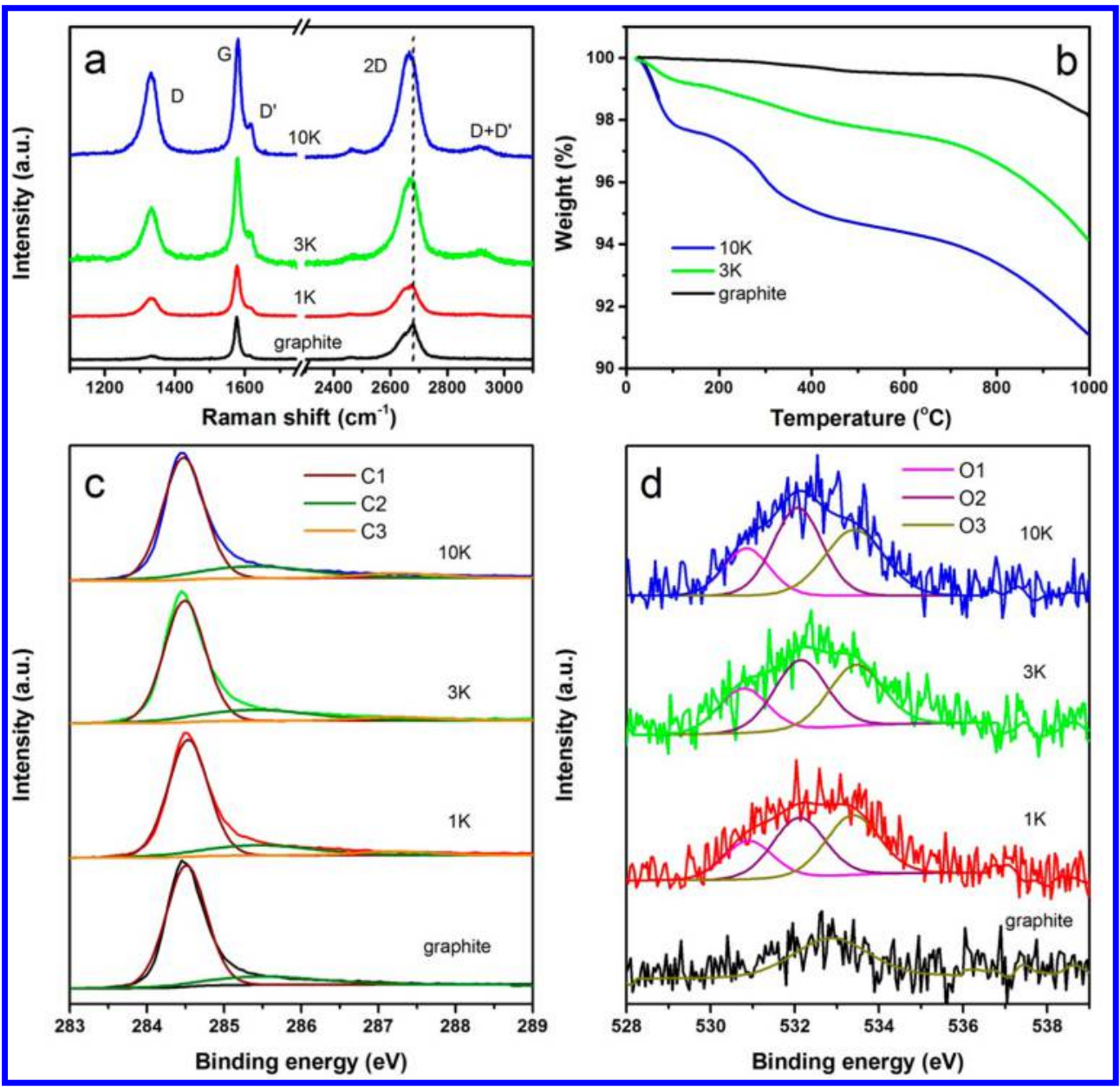

Figure 3. (a) Raman spectra showing increased D band intensity and a $2 \mathrm{D}$ peak downshift with increased symmetry indicating exfoliation and flake size reduction. (b) Thermogravimetric analysis (TGA) plots of graphite, $3 \mathrm{~K}$ and $10 \mathrm{~K}$. Measurements were carried out under $\mathrm{N}_{2}$ atmosphere with a heating rate of $5{ }^{\circ} \mathrm{C}$ min over the temperature range $24-1000{ }^{\circ} \mathrm{C}$. High resolution (c) $\mathrm{C} 1 \mathrm{~s}$ and (d) $\mathrm{O} 1 \mathrm{~s}$ deconvoluted X-ray photoelectron spectroscopy (XPS) of graphite, $1 \mathrm{~K}, 3 \mathrm{~K}$, and $10 \mathrm{~K}$.

interference between electrons scattered from the A-type and B-type layers. Conversely, for a single graphene layer, the intensities of the inner $\{1100\}$-type spots are stronger than those of the $\{2100\}$-type spots. For our materials line profiles extracted along the -2110 to 1-210 axis for the three oriented diffraction patterns were used to identify the thickness of layers along these directions. ${ }^{37,38}$ The ratios of $\{1100\}$ to $\{2110\}$ for all three different orientations are all larger than 1 , indicating that the stacking consists of individual graphene layers along each of the three different directions.

A clear hexagonal arrangement could be seen in the HRTEM images of Figure 2i, revealing that the exfoliated graphene sheets are of high crystalline quality and at the same time confirming that ionic liquid assisted grinding did not damage or disrupt the hexagonal structure of graphene. In Figure 2i, a portion of the image has been digitally noise filtered. The clearly visible parallel lines demonstrate the regular period of the lattice planes. Energy dispersive X-ray analysis (EDAX) of $10 \mathrm{~K}$ product (Figure S6, Supporting Information) confirms the presence of only carbon and oxygen, indicating the product is of high quality with absence of impurities.

The morphologies of the $3 \mathrm{~K}$ and $10 \mathrm{~K}$ catalysts were further confirmed with atomic force microscopy (AFM) measurements. Figure S7 (Supporting Information) is an AFM image of the $3 \mathrm{~K}$ product, where clearly distinguishable layers of both small $(400 \mathrm{~nm})$ and larger $(\sim 1 \mu \mathrm{m})$ lateral dimensions are observed with a variable sheet thicknesses between 2 and $6 \mathrm{~nm}$. Graphene sheets less than 10 layers thick with lateral diameters of a few hundred nanometers were observed for the $10 \mathrm{~K}$ catalyst (Figure S8, Supporting Information).

The progressive exfoliation and reduction in lateral size of the centrifugation products was corroborated by XRD measurements. As can be seen in Figure $2 j$, the pristine graphite exhibited a typical strong peak at $26.52^{\circ}$, corresponding to an interlayer $d$-spacing of $0.3358 \mathrm{~nm}$ of the (002) plane. The centrifugation products displayed a progressive decrease in peak intensity. The $10 \mathrm{~K}$ product retained only $0.5 \%$ of the (002) peak intensity of the pristine graphite flakes and exhibited a dramatic broadening (Table S1, Supporting Information), indicating considerably reduced lateral size. ${ }^{40,41}$ The slight increase in interplanar spacing (from 0.3358 to $0.3378 \mathrm{~nm}$ ) is probably due to the fact that the layers, although aggregated during film formation, are decoupled from each other.

To further evaluate the structure of the graphene nanosheets, Raman spectra were taken on a Labram Raman spectroscope with a laser excitation wavelength of $632.8 \mathrm{~nm}$ and a beam size of approximately $2 \mu \mathrm{m}$. Raman spectroscopy is a powerful tool for identifying the number of layers and structural defects of graphene. $^{42,43}$ Figure $3 a$ shows the evolution of the spectral 
features of the products upon various centrifugation cycles together with that of graphite. Pristine graphite can be seen to display the characteristic Raman 2D signal of graphite, having a double peak structure. It exhibits a small detectable $\mathrm{D}$ band at around $1334 \mathrm{~cm}^{-1}$, and a D-band to G-band intensity ratio $\left(I_{\mathrm{D}} /\right.$ $I_{\mathrm{G}}$ ) of 0.11 . The presence of the small $\mathrm{D}$ band, which is a defect-related peak, most probably is linked with the presence of small flakes, which coexist with the large graphite platelets, as revealed by SEM images, (Figures 1 and S1, Supporting Information). As expected, the ground products produced from the sequential centrifugation showed strong $\mathrm{D}$ bands around $1332 \mathrm{~cm}^{-1}$ with the $I_{\mathrm{D}} / I_{\mathrm{G}}$ ratios in the range of $0.37-0.72$, indicating progressive size reduction, which is consistent with the TEM and SEM results (Figures 1, 2, and S1-S5, Supporting Information). The progressive increase of the D peak suggests that the $\mathrm{D}$ band should arise predominantly from edge defects upon increased centrifugation speeds, rather than basal plane defects, because the nanosheet lateral sizes were typically smaller than the laser spot diameter $(2 \mu \mathrm{m}){ }^{43,44}$ The observed $I_{\mathrm{D}} / I_{\mathrm{G}}$ values are lower than those of large size reduced graphene oxide, where a considerable amount of disorder remains after the reduction of graphene oxide material. ${ }^{45-47}$ The disorder related peaks, $\mathrm{D}^{\prime}$ and $\mathrm{D}+\mathrm{D}^{\prime}$ became apparent for the $3 \mathrm{~K}$ and $10 \mathrm{~K}$ products. The $2 \mathrm{D}$ band becomes more symmetrical with increasing the centrifugation speed and is downshifted from $\sim 2675 \mathrm{~cm}^{-1}$ at $1 \mathrm{~K}$ to $\sim 2666 \mathrm{~cm}^{-1}$ at $10 \mathrm{~K}$. The development of a more symmetrical 2D line shape together with the blue shift of $2 \mathrm{D}$ band relative to graphite, suggests that the $10 \mathrm{~K}$ product consists of few layers. ${ }^{43}$ The results confirm that the deposited nanosheets consist of a disordered arrangement of randomly restacked but weakly interacting few layer graphene nanosheets, in agreement with the TEM observations.

The smaller size and exfoliation under progressive centrifugation steps was also confirmed by Thermogravimetric analysis (TGA). TGA graphs (Figure $3 \mathrm{~b}$ ) of both $3 \mathrm{~K}$ and $10 \mathrm{~K}$ products are presented and compared with that of intact graphite. The latter is thermally stable when heated up to 1000 ${ }^{\circ} \mathrm{C}$ under nitrogen atmosphere, indicated by a weight loss of only $1.90 \%$; however, the $3 \mathrm{~K}$ and $10 \mathrm{~K}$ products show a gradual higher weight loss of 5.8 and $8.9 \%$, respectively, reflecting the smaller lateral dimensions and higher degree of exfoliation. It is expected that a few layer graphene with small dimensions will have lower combustion temperature compared to large area graphitic sheets. ${ }^{48}$ The $10 \mathrm{~K}$ product exhibits a higher weight loss than the rest of the samples due to the presence of a large amount of a few layer nanosized graphene. The graphite exhibits the greatest overall thermal stability due to the extended stacking and large lateral size of graphite platelets and as a result, requires a much higher temperature for decomposition. Our findings are in agreement with the report by Kinoshita et al., ${ }^{49}$ which indicates that thermal decomposition temperatures for natural graphite powders decrease as the density of edge plane sites increase.

Differential scanning calorimetry (DSC) DSC-TGA plots (Figure S9, Supporting Information) show the 10K product typically exhibited two well-defined mass losses, which took place at $25-125{ }^{\circ} \mathrm{C}$ and $140-400{ }^{\circ} \mathrm{C}$, with peaks at 62.6 and $292{ }^{\circ} \mathrm{C}$, respectively. These observed weight losses can be attributed to gasification of adsorbents and entrapped DMF between the graphene layers (boiling point $153{ }^{\circ} \mathrm{C}$ ) as well as the thermal decomposition of oxygen edge groups.
XPS analysis provided explicit information on the nature and exact content of any functional groups brought by the exfoliation. Figure 3c,d and Tables S2-S4 (Supporting Information) summarize the results of the XPS investigation of graphite and centrifugation products. XPS wide spectra from all samples show a strong $\mathrm{C} 1 \mathrm{~s}$ peak at $284.5 \mathrm{eV}$ associated with the $\mathrm{sp}^{2}$ hybridized framework and a minor $\mathrm{O} 1 \mathrm{~s}$ peak at approximately $532.5 \mathrm{eV}$ (Figure S10a, Supporting Information). The high resolution carbon $\mathrm{C} 1 \mathrm{~s}$ (Figure 3c) spectra were fit with up to three peaks using binding energies of 284.47, 285.40, and $287.72 \mathrm{eV}$. These peaks correspond to the following carbon components: $\mathrm{sp}^{2}(\mathrm{C} 1), \mathrm{C}-\mathrm{O}$ associated with ether and phenolic groups $(\mathrm{C} 2)$ and $\mathrm{C}=\mathrm{O}(\mathrm{C} 3)^{50,51}$ groups, respectively. The N 1s, F 1s, P 2p, Co 2p, Co 2p, Fe 2p, Ni 2p, and Mn 2p core level spectra show no observable nitrogen, fluorine, phosphorus, and metal traces (Figure S10b-h, Supporting Information). These results confirm the absence of any functional groups or metals accrued from the ionic liquid, DMF solvents, or grinder. Thus, the ionic liquid used during the grinding process does not bind to the inert basal graphene plane, and is effectively removed to a level below the XPS detection limit. Prolonged sonication in DMF has also been avoided and therefore nitrogen moieties cannot be inherited by DMF. $^{52}$ They also confirm that the grinding process does not introduce any traces of metal impurities in the composition of the products. The $\mathrm{O} 1 \mathrm{~s}$ spectra contain contributions from $\mathrm{O} 1$, oxygen doubly bonded to aromatic carbon $(\mathrm{C}=\mathrm{O})$ at 530.77 $\mathrm{eV}$; O2, singly bonded oxygen to $\mathrm{C}-\mathrm{OH}$ and $\mathrm{C}-\mathrm{O}-\mathrm{C}$ at $532.00 \mathrm{eV}$; O3, singly bonded oxygen $\mathrm{O}=\mathrm{C}-\mathrm{OH}, \mathrm{O}=\mathrm{C}-$ OR at $533.11 \mathrm{eV}^{50,51} \mathrm{An}$ increase of no more than 1.5 at. \% was observed in the oxygen content of the ground centrifugation products compared to that of the starting graphite (starting graphite, $1.08 \pm 0.28$ at. \%; $1 \mathrm{~K}, 2.34 \pm$ 0.26 at. $\% ; 3 \mathrm{~K}, 2.08 \pm 0.50$ at. $\% ; 10 \mathrm{~K}, 2.58 \pm 0.21$ at. $\%)$. This increase is due to the existence of a larger amount of edges, which can be attacked by oxygen in air. Figure S11 (Supporting Information) shows a very good overlap between the $C 1$ s of graphite and $10 \mathrm{~K}$, indicating an identical composition.

Using the inductively coupled plasma mass spectrometry (ICP-MS) technique, we assessed the starting metallic-impurity content of natural graphite as well as the variation of the impurities in the centrifugation products $3 \mathrm{~K}$ and $10 \mathrm{~K}$. Table 1 summarizes the results of the analysis.

Table 1. Impurity Content (ppm) in Starting Graphite, 3K, and 10K Catalysts As Determined by ICP-MS Analysis

\begin{tabular}{lccc} 
& graphite & $3 \mathrm{~K}$ & $10 \mathrm{~K}$ \\
$\mathrm{Mn}$ & 0.66 & 0.28 & 0.14 \\
$\mathrm{Co}$ & 0.25 & 0.28 & 0.39 \\
$\mathrm{Ni}$ & 0.57 & 0.65 & 1.68 \\
$\mathrm{Fe}$ & <detection limit & <detection limit & <detection limit \\
$\mathrm{Mo}$ & 0.45 & 0.42 & 0.03 \\
$\mathrm{P}$ & 6.62 & 65.40 & 70.60 \\
\hline
\end{tabular}

It can be clearly seen from Table 1 that for graphite, $3 \mathrm{~K}$, and $10 \mathrm{~K}$ products, metal impurities ( $\mathrm{Mn}, \mathrm{Co}, \mathrm{Ni}, \mathrm{Fe}, \mathrm{Mo}$ ) are present in concentrations below 2 ppm, which clearly demonstrates that the starting graphite was of high purity and the grinding process did not introduce any metals contaminants to the $3 \mathrm{~K}$ and $10 \mathrm{~K}$ products. A small increase in the phosphorus level from $6.62 \mathrm{ppm}$ in the starting graphite to 


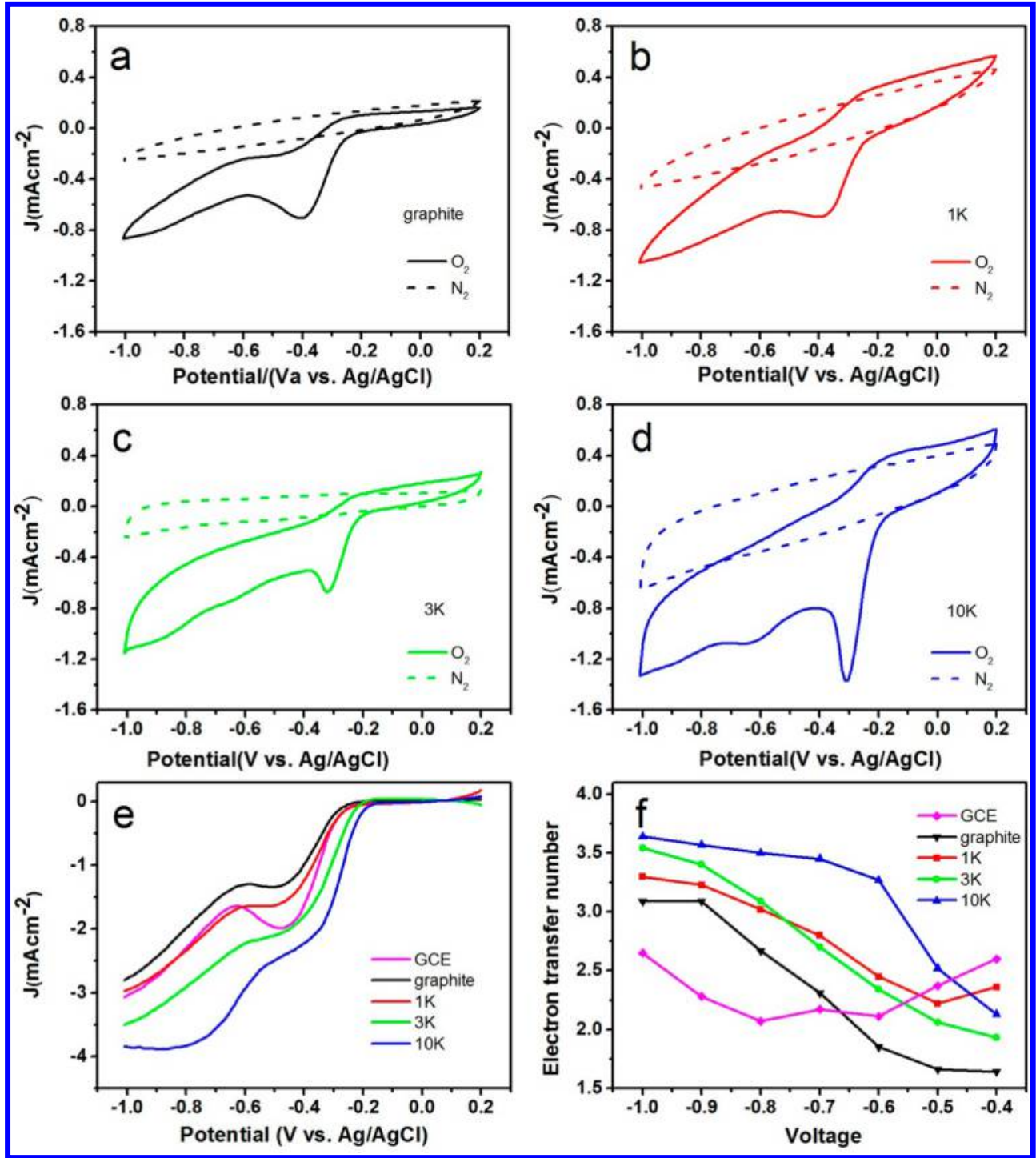

Figure 4. Cyclic voltammograms of catalysts in oxygen or nitrogen saturated $0.1 \mathrm{M} \mathrm{KOH}$ at a scan rate of $100 \mathrm{mV} \mathrm{s}^{-1}$. (a) Pristine graphite. (b) $1 \mathrm{~K}$. (c) $3 \mathrm{~K}$. (d) $10 \mathrm{~K}$. (e) Linear sweep performance at a rotation speed of $1600 \mathrm{rpm}$ of the four catalysts in oxygen saturated $0.1 \mathrm{M} \mathrm{KOH}$ at a scan rate of $10 \mathrm{mV} \mathrm{s}^{-1}$. (f) Electrons transferred per oxygen molecule for the four catalysts over potential range $-0.4 \mathrm{~V}$ to $-1.0 \mathrm{~V}$ based on the Koutecky-Levich equation. For all electrochemical measurements, a $3 \mathrm{~mm}$ diameter BASI GCE was used and a catalyst loading of $0.283 \mathrm{mg} / \mathrm{cm}^{2}$.

70.60 ppm (0.0071 at. \%) in $10 \mathrm{~K}$, was observed, which originates from the ionic liquid.

The ORR electrocatalytic activity of both starting graphite flakes and centrifugation products was first evaluated by cyclic voltammetry $(\mathrm{CV})$ in $\mathrm{N}_{2}$ and $\mathrm{O}_{2}$ saturated $0.1 \mathrm{M}$ aqueous solution of $\mathrm{KOH}$, as shown in Figure $4 \mathrm{a}-\mathrm{d}$. Comparative studies were performed on all catalysts with the same mass loading of $0.283 \mathrm{mg} / \mathrm{cm}^{2}$. Conditioning of the $10 \mathrm{~K}$ catalyst is displayed in Figure S12 (Supporting Information), showing the 1 st and 20th CV scan. Voltammograms without any peaks were obtained in the absence of oxygen for all catalysts. A relatively increased quasirectangular shape was obtained for the $10 \mathrm{~K}$ graphene nanosheets due to the increased surface area associated with their small lateral size and reduced number of layers. When $\mathrm{O}_{2}$ was introduced, a typical cyclic voltammogram of graphite showed one broad reduction peak at $-0.40 \mathrm{~V}$, which is associated with a two-electron electrochemical reduction. In contrast, the $10 \mathrm{~K}$ product showed two reduction peaks located at -0.31 and $-0.66 \mathrm{~V}$, which are associated with two successive two-electron electrochemical reductions. The reduction peak at
$-0.31 \mathrm{~V}$ is attributed to the reduction process of $\mathrm{O}_{2}$ to $\mathrm{HO}_{2}{ }^{-}$, whereas the peak at $-0.66 \mathrm{~V}$ corresponds to the reduction of $\mathrm{HO}_{2}{ }^{-}$to $\mathrm{OH}^{-}$. Similarly, the $3 \mathrm{~K}$ product showed two ORR peaks but of lower intensities centered at more negative potentials of -0.40 and $-0.66 \mathrm{~V}$, respectively, (Figure $4 \mathrm{c}$ ). The main cathodic peak potential for the $10 \mathrm{~K}$ product is more positive than that of various nitrogen doped graphene ${ }^{52,53}$ and close to the cathodic peak of edge-nitrogenated graphene nanoplatelets $(-0.28 \mathrm{~V}) .{ }^{54}$ These results highlight the low overpotential for ORR on nanosized graphene with a highly crystalline basal plane, free from metal impurities and edge functionalized groups with only a low amount of oxygen content.

To gain further insight into the role of abundant edges on the ORR electrochemical process the electrocatalytic performance of the above four catalysts was examined by linear sweep voltammetry $(\mathrm{LSV})$ in an aqueous solution of $\mathrm{O}_{2}$-saturated 0.1 $\mathrm{M} \mathrm{KOH}$, at a rotation rate of $1600 \mathrm{rpm}$ and a scan rate of 10 $\mathrm{mV} / \mathrm{s}$ on a $3 \mathrm{~mm}$ diameter BASI GC electrode. The effect of mass loading for the $10 \mathrm{~K}$ catalyst on LSVs is presented in 
Figure S13 (Supporting Information). LSVs presented in Figure $4 \mathrm{e}$ have been corrected by subtracting the background response obtained in $\mathrm{N}_{2}$ saturated $0.1 \mathrm{M} \mathrm{KOH}$ solution. Compared to graphite, the $1 \mathrm{~K}, 3 \mathrm{~K}$, and $10 \mathrm{~K}$ products are significantly more active in terms of lower overpotential and larger current density (Figures $4 \mathrm{e}$ and S14a, Supporting Information). The LSVs reveal an appreciable change on the onset potential between graphite $(-0.19 \mathrm{~V})$ and centrifugation products $(1 \mathrm{~K},-0.19 ; 3 \mathrm{~K},-0.16 ; 10 \mathrm{~K},-0.13 \mathrm{~V})$ as well as stronger diffusion current densities $\left(10 \mathrm{~K},-3.85 \mathrm{~mA} / \mathrm{cm}^{2}\right)$ through the whole potential range as the centrifugation speed increases. A higher population of edge defects is present on the centrifugation products compared to that on graphite, and their surplus has a substantial effect on the onset potential. Remarkably, the $10 \mathrm{~K}$ electrode exhibited a more positive onset potential and a much larger limiting current for the ORR in $0.1 \mathrm{M} \mathrm{KOH}$ solution, compared to the edge functionalized (with hydrogen, carboxylic acid, sulfonic acid and carboxylic acid/sulfonic acid) graphene nanoplatelets prepared through a ball milling technique ${ }^{55}$ or reduced graphene oxide. ${ }^{56}$ The performance is similar to macroporous graphitic carbon nitride/carbon composite $\left(\mathrm{g}-\mathrm{C}_{3} \mathrm{~N}_{4} / \mathrm{C}\right.$, onset: $-0.14 \mathrm{~V}$; at -1 $\left.\mathrm{V}, J \sim 4 \mathrm{~mA} / \mathrm{cm}^{2}\right),{ }^{57}$ and comparable to that of threedimensional nitrogen-doped graphene aerogel-supported $\mathrm{Fe}_{3} \mathrm{O}_{4}$ nanoparticles $\left(\mathrm{Fe}_{3} \mathrm{O}_{4} / \mathrm{N}\right.$-gas, onset: $-0.19 \mathrm{~V}$; at $-1 \mathrm{~V}, J=4.2$ $\left.\mathrm{mA} / \mathrm{cm}^{2}\right),{ }^{54}$ nitrogen doped graphene infused with iron nanoparticles $\left(\mathrm{NG} / \mathrm{Fe}_{5}\right.$, onset: $-0.04 \mathrm{~V}$; at $-1 \mathrm{~V}, J=4 \mathrm{~mA}$ / $\left.\mathrm{cm}^{2}\right) .55$

These results highlight the improved oxygen reduction ability of the $10 \mathrm{~K}$ catalyst, which is clearly due to the following synergetic effects: (i) the abundance of edges capable to influence the adsorption and dissociation of $\mathrm{O}_{2}$ and (ii) the efficient electron transfer between the electrode and active edge sites ensued from the reduced number of layers and the high structural crystalline quality of the basal plane. It is known that both graphite and a few layer graphene exhibit anisotropic electron conduction in directions parallel and perpendicular to the basal plane. The electron transfer in the vertical direction decreases as the number of layers increases. In thick graphite platelets, even though a rich amount of edge sites are exposed in the direction perpendicular to the basal plane their electrocatalytic performance is poor due to the increased electron path.

Because all the centrifugation products and even graphite have similar oxygen contents, it becomes obvious that the gradually enhanced performance is not caused by the limited oxygen sites, ${ }^{58,59}$ but is the outcome of a simultaneous reduction in the lateral size and progressive reduction in the number of layers. The onset potential of $-0.13 \mathrm{~V}$ vs $\mathrm{Ag} / \mathrm{AgCl}$ and the current density of $-3.85 \mathrm{~mA} / \mathrm{cm}^{2}$ at $-1 \mathrm{~V}$, represent the best ORR performance ever achieved from an undoped carbon based catalyst.

Figure S14b (Supporting Information) shows the RDE voltammograms for the ORR on the $10 \mathrm{~K}$ electrode at various rotation speeds (from 400 to $2025 \mathrm{rpm}$ ). With an increase of the rotation speed, the reduction current increases, which can be explained by shortened diffusion distance at high speeds. The Koutecky-Levich plots $\left(j^{-1}\right.$ vs $\left.\omega^{-1 / 2}\right)$ at different electrode potentials displayed good linearity (Figure S15, Supporting Information), and thus, the slope of these Koutecky-Levich plots could qualitatively give a good prediction on the catalytic activity for $\mathrm{O}_{2}$ reduction. The highest slope was obtained at the $10 \mathrm{~K}$ catalyst electrode, again suggesting its higher catalytic activity for ORR. The electrontransfer numbers ( $n$ ) of the four electrodes at different potentials were calculated according to the slopes of the linear fitted Koutecky-Levich (K-L) plots $\left(\mathrm{J}^{-1}\right.$ vs $\omega^{-1 / 2}$ ) and are presented in Figure 4f. Generally, increasing $n$ values from 1.64 to 3.54 were obtained for graphite, $1 \mathrm{~K}$, and $3 \mathrm{~K}$ nanosheets as the potential became more negative. This observation reveals that peroxide is formed first at low overpotentials, which is then partially reduced further to $-\mathrm{OH}$ at higher overpotentials, during the oxygen reduction process. In contrast, the $10 \mathrm{~K}$ product exhibited much higher $n$ values through the whole potential range compared to graphite, $1 \mathrm{~K}$ and $3 \mathrm{~K}$ products. Based on the $n$ values the electrocatalytic activity was in the order: $10 \mathrm{~K}>3 \mathrm{~K}>1 \mathrm{~K}>$ graphite. Over the potential range $-0.7 \mathrm{~V}$ to $-1 \mathrm{~V}$, the $n$ values for the $10 \mathrm{~K}$ electrode are almost stable (3.6-3.4), indicating that the reduction takes place through an "apparent" four-electron transfer reaction, due to faster hydrogen peroxide decomposition by the electron rich edges. Clearly, our results indicate that a substantial improvement in the electrocatalysis of ORR can be brought by a simultaneous reduction in both the lateral size and number of layers of graphene nanosheets. These changes bring an increase in the number of catalytic active sites and efficient transfer of electrons both of which synergistically enhance the ORR activity.

The reduction of molecular oxygen can proceed through two major pathways under alkaline conditions. A direct fourelectron pathway is the preferred route, producing the desired $\mathrm{OH}^{-}$described by eq 1 . Alternatively, ORR can go through a $2 \mathrm{e}^{-}+2 \mathrm{e}^{-}$pathway involving the production of a peroxide $\mathrm{HO}_{2}{ }^{-}$intermediate in the first $2 \mathrm{e}^{-}$process (eq 2), and subsequently undergo a further $2 \mathrm{e}^{-}$reduction (eq 3 ). ${ }^{60}$ If reactions 2 and 3 are very fast, it would then appear as if oxygen is reduced directly to $\mathrm{OH}^{-}$through the four-electron transfer process.

$$
\begin{aligned}
& \mathrm{O}_{2}+2 \mathrm{H}_{2} \mathrm{O}+4 \mathrm{e}^{-} \rightarrow 4 \mathrm{OH}^{-} \\
& \mathrm{O}_{2}+\mathrm{H}_{2} \mathrm{O}+2 \mathrm{e}^{-} \rightarrow \mathrm{HO}_{2}^{-}+\mathrm{OH}^{-} \\
& \mathrm{HO}_{2}{ }^{-}+\mathrm{H}_{2} \mathrm{O}+2 \mathrm{e}^{-} \rightarrow 3 \mathrm{OH}^{-}
\end{aligned}
$$

For our electrochemical findings, it would appear that for the $10 \mathrm{~K}$ catalyst, the intermediate product of the first $2 \mathrm{e}^{-}$process of the ORR (i.e., first two-electron reduction to hydrogen peroxide at low overpotential) has as much better chance to undergo the second $2 \mathrm{e}^{-}$process compared with the rest of the catalysts examined.

The hydrogen peroxide yield $\left(\mathrm{H}_{2} \mathrm{O}_{2} \%\right)$ and the number of electrons transferred $(n)$ per oxygen molecule in the ORR were also estimated using rotating ring disk electrode (RRDE) measurements (Figure S16, Supporting Information). An agreement between the K-L and the RRDE methods for the four catalysts studied (graphite, $1 \mathrm{~K}, 3 \mathrm{~K}, 10 \mathrm{~K}$ ) was only possible for the $10 \mathrm{~K}$ over the range $-0.6 \mathrm{~V}$ to $-1 \mathrm{~V}$. Due to the absence of a diffusion limiting region in the rest of the catalysts, no agreement between the calculated $n$ numbers by the two methods was observed. It is generally accepted that the RRDE method, which is able to directly determine the amount of peroxide species produced, generates more accurate results. ${ }^{61}$

To evaluate the contributions to the electrochemical activity due to increased density of active sites per surface area, we employ a Tafel analysis in conjunction with electrochemical double layer capacitance measurements. Tafel analysis of the $j-$ 


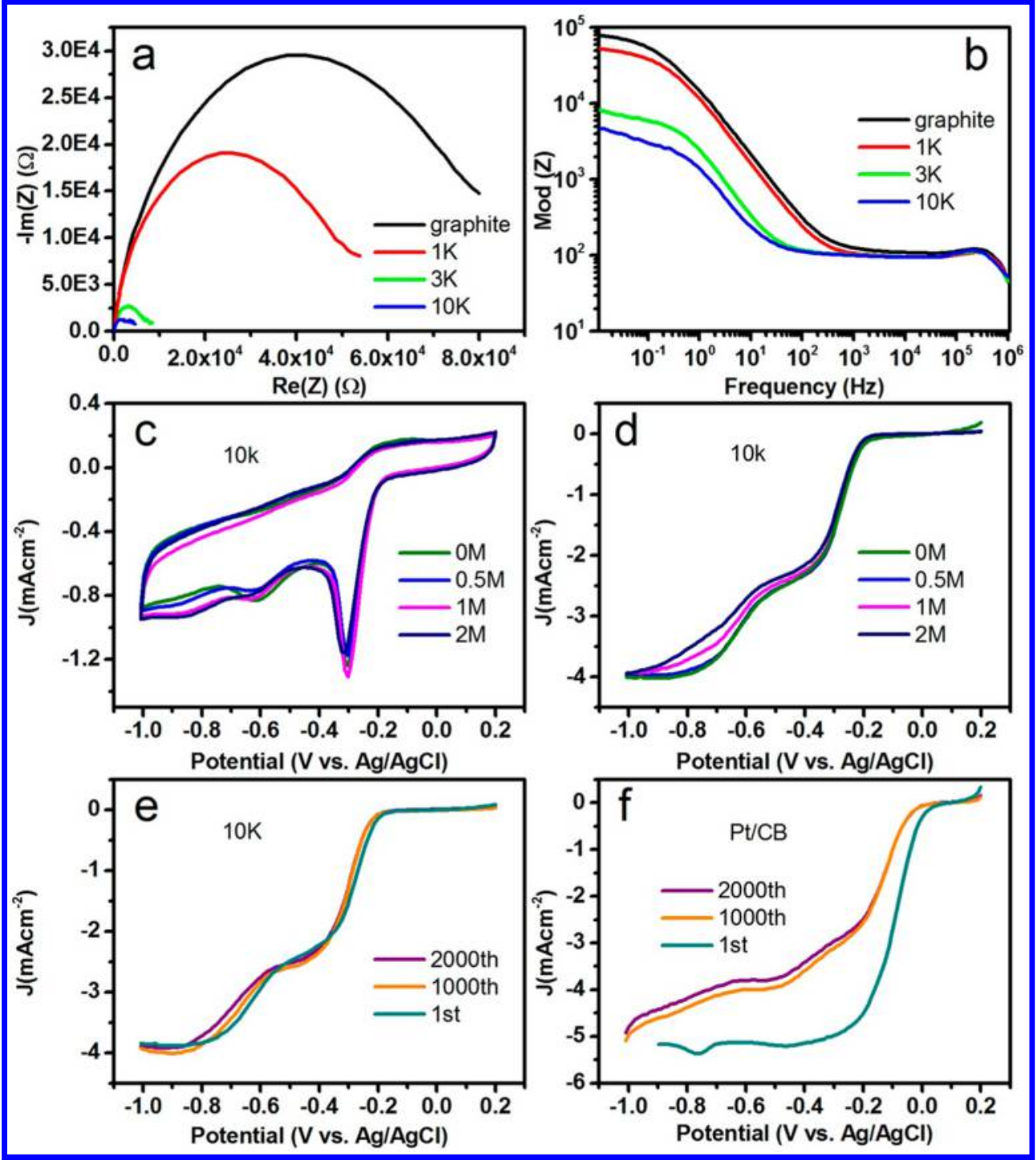

Figure 5. Electrochemical impedance spectroscopy plots in the form of (a) Nyquist and (b) Bode plots of the four catalysts Measurements were performed at $-0.2 \mathrm{~V}$. (c) CV, (d) LS response of $10 \mathrm{~K}$ nanosheets after methanol addition. (e) LS curves of $10 \mathrm{~K}$, (f) benchmark Pt/CB (20 wt \% Pt) after 1st, 1000th, and 2000th CV scans. All measurements were carried out in oxygen saturated 0.1 M KOH using a 3 mm diameter BASI GCE and a catalyst loading of $0.283 \mathrm{mg} / \mathrm{cm}^{2}$.

$v$ curves of the four samples (graphite, $1 \mathrm{~K}, 3 \mathrm{~K}, 10 \mathrm{~K}$ ) exhibited slopes over the range 84 to $77 \mathrm{mV}$ per decade, suggesting a similarity of surface chemistry for the ORR. Comparison of the relative activity of the four samples in terms of the exchange current densities, $j_{0}$ (determined from the extrapolated $x$ intercept where $V=0$ ), revealed the following trend: graphite > $1 \mathrm{~K}>3 \mathrm{~K}>10 \mathrm{~K}$, as seen in Figure S17 (Supporting Information).

The relative electrochemical surface active area of the catalysts was estimated by measuring the electrochemical double-layer capacitance $\left(\mathrm{C}_{\mathrm{DL}}\right)$ over the potential range of -0.1 to $+0.1 \mathrm{~V}$ vs $\mathrm{Ag} / \mathrm{AgCl}$. This potential range contains no faradaic current and is associated with the double layer charging. The charging current, $i_{\mathcal{c}}$ is then measured at various scan rates, $\nu$, between 0.1 and $0.01 \mathrm{~V} \mathrm{~s}^{-1}$, (Figure S18, Supporting Information). The $C_{\mathrm{DL}}$, as given by eq $4 .{ }^{57,62}$

$$
J_{\mathrm{c}}=\nu \cdot C_{\mathrm{DL}}
$$

Thus, a plot of $j_{c}$ vs $\nu$ gives a straight line with a slope equal to the $C_{\mathrm{DL}}$, as shown in Figure S19 (Supporting Information).
The estimated $C_{\mathrm{DL}}$ values are given in Table S5 (Supporting Information). The analysis reveals a $1.6-3.7 \times$ increase in the density of active sites per surface area for the various centrifugation products compared to graphite, enabling the $10 \mathrm{~K}$ to outperform by almost 4 times.

Theoretical calculations have shown carbon atoms in graphene that possess high spin or charge density are the effective electrocatalytic active sites for ORR. ${ }^{19}$ The basal plane of pure graphene does not possess any asymmetry in charge or spin density and therefore does not have catalytic activity for ORR. Recently, functional density calculations have predicted that carbon atoms at the zigzag edge possess high positive charge density, which can act as catalytic active sites for ORR. ${ }^{63}$ Therefore, a plethora of zigzag edges could provide more active sites, where the carbon atoms with high charge density follow a four-electron transfer pathway. This hypothesis is supported by our findings, where abundant edges on the $10 \mathrm{~K}$ nanosized graphene result in enhanced ORR activity. In general, exfoliated graphene flakes appear to show zigzag and armchair edges with comparable probability. ${ }^{25,64}$ Therefore, an increase in the 
overall edge sites would also increase the population of active zigzag edges.

Even though an abundance of zigzag edge sites is beneficial, the layer number should also be optimized. During ORR, the electron transfer has to proceed between the electrode support and the edges of all successive layers, which leads to significantly increased resistance. Resistivity has been measured to be larger vertical to the basal graphene planes as compared to parallel to the planes. ${ }^{65}$ This means that a single layer or a few layer nanosized graphene is the optimal structure compared to a multilayer nanosized graphene, because of the shorter electron path.

This sequence was further confirmed by the electrochemical impedance spectra (EIS), which can be used to probe the interfacial processes and kinetics of electrode reactions in electrochemical systems. EIS measurements were performed at a potential $-0.2 \mathrm{~V}$ for the four catalysts. The Nyquist plots, shown in Figure 5a, which present impedance values in the form of imaginary $\left(Z_{\mathrm{Im}}\right)$ vs real $\left(Z_{\mathrm{Re}}\right)$ parts at various frequencies, showed an arc-like profile for all four catalysts. As graphite possesses a low ORR activity, this is mirrored with a large charge transfer resistance, as it does not allow fast shuttling of electrons during ORR. The $10 \mathrm{~K}$ exhibits the smallest semicircle, which clearly demonstrates that the $10 \mathrm{~K}$ electrode possesses significantly lower charge transfer resistance and thus allows a much faster transport of electrons during ORR. The overall magnitude of the impedance in the low frequency range, as determined by the intercept of the straight lines with the vertical axis (modulus of $Z$ ) in the Bode plot shown in Figure $5 \mathrm{~b}$, follows the order graphite $>1 \mathrm{~K}>3 \mathrm{~K}>$ $10 \mathrm{~K}$. We rationalize the larger decrease in impedance for $10 \mathrm{~K}$ in terms of its earlier onset potential and hence higher catalytic activity.

Tolerance to crossover has been investigated through the introduction of methanol to the $\mathrm{O}_{2}$ saturated $\mathrm{KOH}$ electrolyte. Both graphite and $10 \mathrm{~K}$ product demonstrate very little alteration in both $\mathrm{CV}$ responses and LS voltammograms compared to commercial platinum carbon black (Pt/CB) upon introduction of various concentrations of methanol, as shown in Figures 5c,d and S20 (Supporting Information). This demonstrates their high catalytic selectivity for the ORR against methanol oxidation. The ORR activities of starting graphite, $10 \mathrm{~K}$, and $\mathrm{Pt} / \mathrm{CB}\left(0.283 \mathrm{mg} / \mathrm{cm}^{2}\right)$ before and after 2000 cycles are shown in Figures S21 (Supporting Information) and $5 \mathrm{e}, \mathrm{f}$, respectively. There are only $15 \mathrm{mV}$ are $24 \mathrm{mV}$ shifts of the first half-wave potentials $\left(E_{1 / 2}\right)$ for graphite and $10 \mathrm{~K}$, respectively. By comparison, the shift of $E_{1 / 2}$ is $107 \mathrm{mV}$ for commercial $\mathrm{Pt} / \mathrm{CB}$ after 2000 cycles, revealing the superior durability of the $10 \mathrm{~K}$ product.

Through this study, by investigating progressively thinner and smaller graphene nanosheets with minimal oxygen content and no metal contamination, new insights are gained into the roles of graphene edges and thickness on the ORR activity. Experimentally, it was shown that the lateral size reduction of few layer graphene effectively elevates the electrode onset potential and current density, resulting in enhancement of the ORR activity. There is currently an increasing notion that the activity and reaction kinetics for the ORR are strongly correlated with the work function (WF) of the electrocatalyst. $^{66,67} \mathrm{~A}$ decrease in WF is associated with an enhancement in ORR activity. Theoretical studies predict and experimental studies have verified that that the WF of graphene is in a similar range to that of graphite, $\sim 4.6 \mathrm{eV}^{68}$ however, it depends sensitively with the number of layers and lateral size. ${ }^{68-70}$ It has been predicted that the WF decreases with a reduction in the number of layers and lateral size. Therefore, the progressive enhancement in ORR for the graphene products can be explained through a progressive decrease in the WF.

\section{CONCLUSION}

We have developed a simple and versatile ionic liquid assisted grinding process coupled with sequential centrifugation steps to efficiently exfoliate the pristine graphite directly into a few layer nanosized graphene with low oxygen content $(<2.5 \pm 0.2$ at. $\%)$. We have examined for the first time their catalytic activity for oxygen reduction reaction. We found that the nanosized graphene exhibits an enhanced ORR performance, which is attributed to the synergy of two main factors (i) the abundance of edges sites accrued from the small lateral size and (ii) the efficient electron transfer between the active edge sites and the electrode. The last was ensued from the reduced number of graphene layers and the high structural crystalline quality of their basal plane. The progressive increase in ORR activity is a clear indication that the improvement is merely due to the size and thickness reduction and not an artifact from metal contamination or functional groups. The approach presented herein is applicable to the synthesis of other 2D nanomaterials. The size and thickness dependent catalytic activity of graphene nanosheets has important implications in understanding and further improving the catalytic activities of metal free graphene and possibly other $2 \mathrm{D}$ nanomaterials. Although the ORR performance is not comparable to commercial $\mathrm{Pt} / \mathrm{CB}$ catalysts, this work could offer opportunities for stimulating future experimental and theoretical research on the role of sheet size and number of layers of pristine graphene on electrocatalysis.

\section{ASSOCIATED CONTENT}

\section{S Supporting Information}

Synthesis of graphene products, electrochemical measurements, and characterisation methods. This material is available free of charge via the Internet at http://pubs.acs.org.

\section{AUTHOR INFORMATION}

\section{Corresponding Author}

*P. Papakonstantinou. E-mail: p.papakonstantinou@ulster.ac. uk.

\section{Notes}

The authors declare no competing financial interest.

\section{ACKNOWLEDGMENTS}

This work was financially supported by a Leverhulme Trust/ Royal Academy of Engineering Senior Research Fellowship to P.P.; a Ph.D. Studentship to J.B. from the Department of Employment and Learning in Northern Ireland; a visiting Senior Research Fellowship to M.L. by the University of Ulster; The Chinese Thousand-Talents plan program and the Jiangsu Shuangchuang program (Q.X. and P.W.).

\section{REFERENCES}

(1) Steele, B. C. H.; Heinzel, A. Materials for Fuel-Cell Technologies. Nature 2001, 414, 345-352.

(2) Debe, M. K. Electrocatalyst Approaches and Challenges for Automotive Fuel Cells. Nature 2012, 486, 43-51.

(3) Gasteiger, H. A.; Markovic, N. M. Just a Dream - or Future Reality? Science 2009, 324, 48-49. 
(4) Girishkumar, G.; McCloskey, B.; Luntz, A. C.; Swanson, S.; Wilcke, W. Lithium-Air Battery: Promise and Challenges. J. Phys. Chem. Lett. 2010, 1, 2193-2203.

(5) Lee, J. S.; Tai Kim, S.; Cao, R.; Choi, N. S.; Liu, M.; Lee, K. T.; Cho, J. Metal-Air Batteries with High Energy Density: Li-Air versus Zn-Air. Adv. Energy Mater. 2011, 1, 34-50.

(6) Chen, Z.; Higgins, D.; Yu, A.; Zhang, L.; Zhang, J. A Review on Non-Precious Metal Electrocatalysts for PEM Fuel Cells. Energy Environ. Sci. 2011, 4, 3167-3192.

(7) Jaouen, F.; Proietti, E.; Lefevre, M.; Chenitz, R.; Dodelet, J.-P.; Wu, G.; Chung, H. T.; Johnston, C. M.; Zelenay, P. Recent Advances in Non-Precious Metal Catalysis for Oxygen-Reduction Reaction in Polymer Electrolyte Fuel Cells. Energy Environ. Sci. 2011, 4, 114-130. (8) Stamenkovic, V. R.; Mun, B. S.; Arenz, M.; Mayrhofer, K. J. J.; Lucas, C. A.; Wang, G.; Ross, P. N.; Markovic, N. M. Trends in Electrocatalysis on Extended and Nanoscale Pt-Bimetallic Alloy Surfaces. Nat. Mater. 2007, 6, 241-247.

(9) Su, D. S.; Zhang, J.; Frank, B.; Thomas, A.; Wang, X.; Paraknowitsch, J.; Schlögl, R. Metal-Free Heterogeneous Catalysis for Sustainable Chemistry. ChemSusChem 2010, 3, 169-180.

(10) Zheng, Y.; Jiao, Y.; Jin, Y.; Jaroniec, M.; Qiao, S. Z. Nanostructured Metal-Free Electrochemical Catalysts for Highly Efficient Oxygen Reduction. Small 2012, 8, 3550-3566.

(11) Huang, C.; Li, C.; Shi, G. Graphene based Catalysts. Energy. Environ. Sci. 2012, 5, 8848-8868.

(12) He, C.; Zhang, J. J.; Shen, P. K. Nitrogen-Self Doped Graphene based Non-Precious Metal Catalyst with Superior Performance to Pt/ CB Catalyst Towards Oxygen Reduction Reaction. J. Mater. Chem. A 2014, 2, 3231-3236.

(13) Qu, L.; Liu, Y.; Baek, J. B.; Dai, L. Nitrogen-Doped Graphene as Efficient Metal-Free Electrocatalyst for Oxygen Reduction in Fuel Cells. ACS Nano 2010, 4, 1321-1326.

(14) Geng, D.; Chen, Y.; Chen, Y.; Li, Y.; Li, R.; Sun, X.; Ye, S.; Knights, S. Energy's Thirst for Water in China. Energy Environ. Sci. 2011, 4, 11760-11764.

(15) Yang, Z.; Yao, Z.; Li, G.; Fang, G.; Nie, H.; Liu, Z.; Zhou, X.; Chen, X.; Huang, S. Sulfur-Doped Graphene as an Efficient Metal-free Cathode Catalyst for Oxygen Reduction. ACS Nano 2012, 6, 205-211.

(16) Paraknowitsch, J. P.; Thomas, A. Doping Carbons beyond Nitrogen: An Overview of Advanced Heteroatom Doped Carbons with Boron, Sulfur, and Phosphorus for Energy Applications. Energy Environ. Sci. 2013, 6, 2839-2855.

(17) Zheng, Y.; Jiao, Y.; Ge, L.; Jaroniec, M.; Qiao, S. Z. Two-Step Boron and Nitrogen Doping in Graphene for Enhanced Synergistic Catalysis. Angew. Chem., Int. Ed. 2013, 52, 3110-3116.

(18) Liang, J.; Jiao, Y.; Jaroniec, M.; Qiao, S. Z. Sulfur and Nitrogen Dual-Doped Mesoporous Graphene Electrocatalyst for Oxygen Reduction with Synergistically Enhanced Performance. Angew. Chem., Int. Ed. 2012, 51, 11496-11500.

(19) Zhang, L.; Xia, Z. Mechanisms of Oxygen Reduction Reaction on Nitrogen-Doped Graphene for Fuel Cells. J. Phys. Chem. C 2011, $115,11170-11176$.

(20) Wang, S.; Zhang, L.; Xia, Z.; Roy, A.; Chang, D. W.; Baek, J.-B.; Dai, L. BCN Graphene as Efficient Metal-free electrocatalysts for the Oxygen Reduction Reaction. Angew. Chem., Int. Ed. 2012, 51, 42094212

(21) Nakada, K.; Fujita, M.; Dresselhaus, G.; Dresselhaus, M. S. Edge State in Graphene Ribbons: Nanometer Size Effect and Edge Shape Dependence. Phys. Rev. B 1996, 54, 17954-17961.

(22) Fujii, S.; Ziatdinov, M.; Ohtsuka, M.; Kusakabe, K.; Kiguchi, M.; Enoki, T. Role of Edge Geometry and Chemistry in the Electronic Properties of Graphene Nanostructures. Faraday discuss. 2014, DOI: $10.1039 / C 4 F D 00073 \mathrm{~K}$.

(23) Yuan, W.; Zhou, Y.; Li, Y.; Peng, H.; Zhang, J.; Liu, Z.; Dai, L.; Shi, G. The Edge and Basal Plane Specific Electrochemistry of a Single Layer Graphene Sheet. Sci. Rep. 2013, 3, 2248.

(24) Geim, A. K.; Novoselov, K. S. The Rise of Graphene. Nat. Mater. 2007, 6, 183-191.
(25) Neubeck, S.; You, Y. M.; Ni, Z. H.; Blake, P.; Shen, Z. X.; Geim, A. K.; Novoselov, K. S. Direct Determination of the Crystallographic Orientation of Graphene Edges by Atomic Resolution Imaging. Appl. Phys. Lett. 2010, 97, 053110.

(26) Ritter, K. A.; Lyding, J. W. The Influence of Edge Structure on the Electronic Properties of Graphene Quantum Dots and Nanoribbons. Nat. Mater. 2009, 8, 235-242.

(27) Wang, D. W.; Su, D. Heterogeneous Nanocarbon Materials for Oxygen Reduction Reaction. Energy Environ. Sci. 2014, 7, 576-591.

(28) Ji, X.; Banks, C. E.; Crossley, A.; Compton, R. G. Oxygenated Edge Plane Sites Slow the Electron Transfer of the Ferro-/ Ferricyanide Redox Couple at Graphite Electrodes. ChemPhysChem 2006, 7, 1337-1344.

(29) Shang, N. G.; Papakonstantinou, P.; Sharma, S.; Lubarsky, G.; Li, M.; McNeill, D. W.; Quinn, A. J.; Zhou, W.; Blackley, R. Controllable Selective Exfoliation of High-Quality Graphene Nanosheets and Nanodots by Ionic Liquid Assisted Grinding. Chem. Commun. 2012, 48, 1877-1879.

(30) Fukushima, T.; Kosaka, A.; Ishimura, Y.; Yamamoto, T.; Takigawa, T.; Ishii, N. Molecular Ordering of Organic Molten Salts Triggered by Single-Walled Carbon Nanotubes. Science 2003, 300, 2072-2074.

(31) Fukushima, T.; Aida, T. Ionic Liquids for Soft Functional Materials with Carbon Nanotubes. Chem.-Eur. J. 2007, 13, 50485058

(32) Ambrosi, A.; Chua, C. K.; Khezri, B.; Sofer, Z.; Webster, R. D.; Pumera, M. Chemically Reduced Graphene contains Inherent Metallic Impurities Present in Parent Natural and Synthetic Graphite. Proc. Natl. Acad. Sci. U. S. A. 2012, 109, 12899.

(33) Ambrosi, A.; Chee, S. Y.; Khezri, B.; Webster, R. D.; Sofer, Z.; Pumera, M. Metallic Impurities in Graphenes Prepared from Graphite Can Dramatically Influence Their Properties. Angew. Chem., Int. Ed. 2012, 51, 500-503.

(34) Han, M. Y.; Özyllmaz, B.; Zhang, Y.; Kim, P. Energy Band-Gap Engineering of Graphene Nanoribbons. Phys. Rev. Lett. 2007, 98, 206805.

(35) Wang, T.; Gao, D.; Zhuo, J.; Zhu, Z.; Papakonstantinou, P.; Li, Y.; Li, M. Size Dependent Enhancement of Electrocatalytic OxygenReduction and Hydrogen-Evolution Performance of $\mathrm{MoS}_{2}$ Particles. Chemistry.-Eur. J. 2013, 19, 11939-11948.

(36) Long, D.; Li, W.; Ling, L.; Miyawaki, J.; Mochida, I.; Yoon, S. H. Preparation of Nitrogen-Doped Graphene Sheets by a Combined Chemical and hydrothermal Reduction of Graphene Oxide. Langmuir 2010, 26, 16096-16102.

(37) Meyer, J. C.; Geim, A. K.; Katsnelson, M. I.; Novoselov, K. S.; Obergfell, D.; Roth, S.; Girit, C.; Zettl, A. On the Roughness of Singleand Bi-Layer Graphene Membranes. Solid State Commun. 2007, 143, $101-109$.

(38) Horiuchi, S.; Gotou, T.; Fujiwara, M.; Sotoaka, R.; Hirata, M.; Kimoto, K.; Asaka, T.; Yokosawa, T.; Matsui, Y.; Watanabe, K.; Sekita, M. Carbon Nanofilm with a New Structure and Property. Jpn. J. Appl. Phys. 2003, 42, 1073-1076.

(39) Meyer, J. C.; Geim, A. K.; Katsnelson, M. I.; Novoselov, K. S.; Booth, T. J.; Roth, S. The Structure of Suspended Graphene Sheets. Nature 2007, 446, 60-63.

(40) Jeon, I. Y.; Choi, H.-J.; Choi, M.; Seo, J.-M.; Jung, S.-M.; Kim, M.-J.; Zhang, S.; Zhang, L.; Xia, Z.; Dai, L.; Park, N.; Baek, J. B. Facile, Scalable Synthesis of Edge-Halogenated Graphene Nanoplatelets as Efficient Metal-Free Electrocatalysts for Oxygen Reduction Reaction. Sci. Rep. 2013, 3, 1810.

(41) Jeon, I.-Y.; Zhang, S.; Zhang, L.; Choi, H.-J.; Seo, J.-M.; Xia, Z.; Dai, L.; Baek, J. B. Edge-Selectively Sulfurized Graphene Nanoplatelets as Effient Metal Free Electrocatalysts for Oxygen Reduction Reaction: The Electron Spin Effect. Adv. Mater. 2013, 25, 6138-6145.

(42) Dresselhaus, M. S.; Jorio, A.; Hofmann, M.; Dresselhaus, G.; Saito, R. Perspectives on Carbon Nanotubes and Graphene Raman Spectroscopy. Nano Lett. 2010, 10, 751-758.

(43) Ferrari, A. C.; Meyer, J. C.; Scardaci, V.; Casiraghi, C.; Lazzeri, M.; Mauri, F.; Piscanec, S.; Jiang, D.; Novoselov, K. S.; Roth, S.; Geim, 
A. K. Raman Spectrum of Graphene and Graphene Layers. Phys. Rev. Lett. 2006, 97, 187401.

(44) Casiraghi, C.; Hartschuh, A.; Qian, H.; Piscanec, S.; Georgi, C.; Novoselov, K. S.; Basko, D. M.; Ferrari, A. C. Raman spectroscopy of Graphene Edges. Nano Lett. 2009, 9, 1433-1441.

(45) Mattevi, C.; Eda, G.; Agnoli, S.; Miller, S.; Mkhoyan, K. A.; Celik, O.; Mastrogiovanni, D.; Granozzi, G.; Garfunkel, E.; Chhowalla, M. Evolution of Electrical, Chemical, and Structural Properties of Transparent and Conducting Chemically Derived Graphene Thin Films. Adv. Funct. Mater. 2009, 19, 2577.

(46) Su, C. Y.; Xu, Y.; Zhang, W.; Zhao, J.; Tang, X.; Tsai, C. H.; Li, L. J. Electrical and Spectroscopic Characterization of Ultra-Large Reduced Graphene Oxide Monolayers. Chem. Mater. 2009, 21, 56745680.

(47) Wang, H.; Robinson, J. T.; Li, X.; Dai, H. Solvothermal Reduction of Chemically Exfoliated Graphene Sheets. J. Am. Chem. Soc. 2009, 131, 9910-9911.

(48) Welham, N. J.; Williams, J. S. Extended Milling of Graphite and Activated Carbon. Carbon. 1998, 36, 1309-1315.

(49) Jiang, W.; Nadeau, G.; Zaghib, K.; Kinoshita, K. Thermal Analysis of the Oxidation of Natural Graphite-Effect of Particle Size. Thermochim. Acta 2000, 351, 85-93.

(50) Ganguly, A.; Sharm, S.; Papakonstantinou, P.; Hamilton, J. Probing the Thermal Deoxygenation of Graphene Oxide Using HighResolution in Situ X-ray based Spectroscopies. J. Phys. Chem. C 2011, 115, 17009-17019.

(51) Li, H. Determination of Oxygen Functionality on Highly Oriented Pyrolytic Graphite (HOPG). Ph.D. Thesis, Freie University, Berlin, December 2012

(52) Skaltsas, T.; Ke, X.; Bittencourt, C.; Tagmatarchis, N. Ultrasonication Induces Oxygenated Species and Defects onto Exfoliated Graphene. J. Phys. Chem. C 2013, 117, 23272-23278.

(53) Zhang, Y.; Ge, J.; Wang, L.; Wang, D.; Ding, F.; Tao, X.; Chen, W. Manageable N-Doped Graphene for High Performance Oxygen Reduction Reaction. Sci. Rep. 2013, 3, 2771.

(54) Sun, Y.; Li, C.; Shi, G. Nanoporous Nitrogen Doped Carbon Modified Graphene as Electrocatalyst for Oxygen Reduction. J. Mater. Chem. 2012, 22, 12810-12816.

(55) Wu, Z. S.; Yang, S.; Sun, Y.; Parvez, K.; Feng, X.; Müllen, K. 3D Nitrogen-Doped Graphene Aerogel-Supported $\mathrm{Fe}_{3} \mathrm{O}_{4}$ Nanoparticles as Efficient Electrocatalysts for the Oxygen Reduction Reaction. J. Am. Chem. Soc. 2012, 134, 9082-9085.

(56) Parvez, K.; Yang, S.; Hernandez, Y.; Winter, A.; Turchanin, A.; Feng, X.; Müllen, K. Nitrogen-Doped Graphene and its Iron-Based Composite as Efficient Electrocatalysts for Oxygen Reduction Reaction. ACS Nano 2012, 6, 9541-9550.

(57) Deng, D. H.; Yu, L.; Pan, X. L.; Wang, S.; Chen, X. Q.; Hu, P.; Sun, L. X.; Bao, X. H. Size Effect of Graphene on Electrocatalytic Activation of Oxygen. Chem. Commun. 2011, 47, 10016-10018.

(58) McCrory, C. C. L.; Jung, S.; Peters, J. C.; Jaramillo, T. F. Benchmarking Heterogeneous Electrocatalysts for the Oxygen Evolution Reaction. J. Am. Chem. Soc. 2013, 135, 16977-16987.

(59) Nakada, K.; Fujita, M.; Dresselhaus, G.; Dresselhaus, M. S. Edge State in Graphene Ribbons: Nanometer Size Effect and Edge Shape Dependence. Phys. Rev. B 1996, 54, 17954-17961.

(60) Choi, C. H.; Lim, H.-K.; Chung, M. W.; Park, J. C.; Shin, H.; Kim, H.; Woo, S. I. Long-Range Electron Transfer over Graphenebased Catalysts for High-Performing Oxygen Reduction Reactions: Importance of Size, N-Doping and Metallic Impurities. J. Am. Chem. Soc. 2014, 136, 9070-9077.

(61) Li, F.; Chen, Z. Graphene-based Materials as Nanocatalysts [Online]. In Graphene Chemistry: Theoretical Perspective; Jiang, D., Chen, Z., Eds.; John Wiley \& Sons: New York, 2013; Chapter 15, pp 347-349. http://eu.wiley.com/WileyCDA/WileyTitle/productCd1119942128.html\# (accessed October 2, 2014).

(62) Yu, H. Recent Advances in Photoresponsive Liquid-Crystalline Polymers Containing Azobenzene Chromophores. J. Mater. Chem. A 2014, 2, 3047-3054.
(63) Benck, J. D.; Chen, Z.; Kuritzky, L. Y.; Forman, A. J.; Jaramillo, T. F. Amorphous Molybdenum Sulfide Catalysts for Electrochemical Hydrogen Production: Insights into the Origin of their Catalytic Activity. ACS Catal. 2012, 9, 1916-1923.

(64) Zhang, L.; Niu, J.; Li, M.; Xia, Z. Catalytic Mechanism of SulfurDoped Graphene as Efficient Oxygen Reduction Reaction Catalysts for Fuel Cells. J. Phys. Chem. C 2014, 118, 3545-3553.

(65) Tian, J.; Cao, H.; Wu, W.; Yu, Q.; Chen, Y. P. Direct Imaging of Graphene Edges: Atomic Structure and Electronic Scattering. Nano Lett. 2011, 11, 3663-3668.

(66) Kaye, G. W. C.; Laby, T. H. Tables of Physical and Chemical Constants and Some Mathematical Functions, 14th ed.; Longman: London, 1973.

(67) Cheon, J. Y.; Kim, J. H.; Kim, J. H.; Goddeti, K. C.; Park, J. Y.; Joo, S. H. Intrinsic Relationship between Enhanced Oxygen Reduction Reaction Activity and Nanoscale Work Function of Doped Carbons. J. Am. Chem. Soc. 2014, 136, 8875-8878.

(68) Takahashi, T.; Tokailin, H.; Sagawa, T. Tuning the Graphene Work Function by Electric Field Effect. Phys. Rev. B 1985, 32, 83178324.

(69) Giovannetti, G.; Khomyakov, P. A.; Brocks, G.; Karpan, V. M.; van den Brink, J.; Kelly, P. J. Doping Graphene with Metal Contacts. Phys. Rev. Lett. 2008, 101, 026803.

(70) Hibino, H.; Kageshima, H.; Kotsugi, M.; Maeda, F.; Guo, F.-Z.; Watanabe, Y. Dependence of Electronic Properties of Epitaxial FewLayer Graphene on the Number of Layers Investigated by Photoelectron Emission Microscopy. Phys. Rev. B 2009, 79, 125437. 